\title{
Genome-wide survey of putative Serine/Threonine protein kinases in cyanobacteria
}

\author{
Xiaowen Zhang ${ }^{\dagger 1,2}$, Fangqing Zhao ${ }^{\dagger 1,2}$, Xiangyu Guan³, Yu Yang1,2, \\ Chengwei Liang ${ }^{1,2}$ and Song Qin*1
}

\begin{abstract}
Address: ${ }^{1}$ Institute of Oceanology, Chinese Academy of Sciences, Nanhai Road, Qingdao, China, ${ }^{2}$ Graduate University, Chinese Academy of Sciences, Yuquan Road, Beijing, China and ${ }^{3}$ Ocean University of China, Yushan Road, Qingdao, China

Email: Xiaowen Zhang - fwzxw01@yahoo.com.cn; Fangqing Zhao - biofqzhao@gmail.com; Xiangyu Guan - xiangyu_guan@163.com; Yu Yang - yangyu@ms.qdio.ac.cn; Chengwei Liang - liangchw117@yahoo.com.cn; Song Qin* - sqin@ms.qdio.ac.cn

* Corresponding author †Equal contributors
\end{abstract}

Published: 30 October 2007

BMC Genomics 2007, 8:395 doi:10.1186/147I-2164-8-395
Received: 15 February 2007

Accepted: 30 October 2007

This article is available from: http://www.biomedcentral.com/I47I-2/64/8/395

(C) 2007 Zhang et al; licensee BioMed Central Ltd.

This is an Open Access article distributed under the terms of the Creative Commons Attribution License (http://creativecommons.org/licenses/by/2.0), which permits unrestricted use, distribution, and reproduction in any medium, provided the original work is properly cited.

\begin{abstract}
Background: Serine/threonine kinases (STKs) have been found in an increasing number of prokaryotes, showing important roles in signal transduction that supplement the well known role of two-component system. Cyanobacteria are photoautotrophic prokaryotes able to grow in a wide range of ecological environments, and their signal transduction systems are important in adaptation to the environment. Sequence information from several cyanobacterial genomes offers a unique opportunity to conduct a comprehensive comparative analysis of this kinase family. In this study, we extracted information regarding Ser/Thr kinases from 21 species of sequenced cyanobacteria and investigated their diversity, conservation, domain structure, and evolution.
\end{abstract}

Results: 286 putative STK homologues were identified. STKs are absent in four Prochlorococcus strains and one marine Synechococcus strain and abundant in filamentous nitrogen-fixing cyanobacteria. Motifs and invariant amino acids typical in eukaryotic STKs were conserved well in these proteins, and six more cyanobacteria- or bacteria-specific conserved residues were found. These STK proteins were classified into three major families according to their domain structures. Fourteen types and a total of $|3|$ additional domains were identified, some of which are reported to participate in the recognition of signals or substrates. Cyanobacterial STKs show rather complicated phylogenetic relationships that correspond poorly with phylogenies based on $16 \mathrm{~S}$ rRNA and those based on additional domains.

Conclusion: The number of STK genes in different cyanobacteria is the result of the genome size, ecophysiology, and physiological properties of the organism. Similar conserved motifs and amino acids indicate that cyanobacterial STKs make use of a similar catalytic mechanism as eukaryotic STKs. Gene gain-and-loss is significant during STK evolution, along with domain shuffling and insertion. This study has established an overall framework of sequence-structure-function interactions for the STK gene family, which may facilitate further studies of the role of STKs in various organisms. 


\section{Background}

Cyanobacteria, dating back 2.5-3.5 billion years and constituting a single but large taxonomic and phylogenetic group within the domain Eubacteria [1], are characterized by their ability to carry out oxygenic photosynthesis. Moreover, fossilized cyanobacteria appear similar in form to extant species [2]. Cyanobacteria have a pronounced variation in genome size from $1.6 \mathrm{Mb}$ to $9.2 \mathrm{Mb}$ and exhibit extraordinary diversity in terms of morphology and cell activity. They also exhibit the widest range of diversity in ecological habitats of all photosynthetic organisms, including environments that are extremely hot, extremely cold, alkaline and acidic, marine, freshwater, saline, terrestrial, and symbiotic [3]. Prochlorococcus marinus, which has the smallest genome size and can be divided into two distinct ecotypes (high-light adapted and low-light adapted), is the dominant photosynthetic prokaryote in the open ocean [4]. The diazotrophic filamentous cyanobacteria have the largest genome sizes and include strains isolated from fresh water (Anabaena PCC7120), from a plant-cyanobacterial symbionsis (Nostoc punctiforme PCC73102), or from tropical and subtropical oceans (Trichodesmium erythraeum IMS101). Crocosphaera, a novel genus of marine unicellular diazotrophic cyanobacterium, and Gloeobacter, a rod-shaped unicellular cyanobacterium isolated from calcareous rocks, have larger genome sizes $(6.3 \mathrm{Mb}$ and $4.6 \mathrm{Mb})$ than other unicellular cyanobacteria.

The diversity of cyanobacteria is also reflected in the complexity of their signal transduction systems. To cope with changing environmental conditions, cyanobacteria have developed a variety of adaptive mechanisms to respond to external or internal changes. Two-component signal transduction systems, characterized by the transfer of phosphate by a sensor kinase from a His residue on the enzyme to an Asp residue on the response regulator, are widely distributed among bacteria [5,6]. One-component systems, defined as proteins that contain known or predicted input and output domains in a single protein molecule but lack histidine kinase and receiver domains, are considered to be the pre-eminent mechanism for signal transduction in bacteria and archaea, except for cyanobacteria [7]. In contrast, the Ser/Thr-specific protein kinases (STKs) serve as the backbone of the eukaryotes transduction network. However, with the first identification of an STK in Myxococcus xanthus in 1991 [8], regulatory STKs have been repeatedly identified in prokaryotes. Protein phosphorylation on serine/threonine residues in cyanobacteria was first revealed by radioactive labeling of proteins in 1994 [9]. Numerous bacterial STK genes have since been predicted within genome sequences [10-12], and they have been associated primarily with three different processes, namely regulation of development, stress responses, and pathogenicity.
According to Hanks and Hunter, canonical Ser/Thr protein kinases contain 12 conserved subdomains [13] that fold into a common catalytic core structure, as revealed by the 3-dimensional structures of several protein-serine kinases. These 12 conserved sequence motifs, about 280 amino acids in length, are named as subdomains I-V, VIa, VIb and VII-XI $[13,14]$. There are three structural subdomains with separate roles: $\mathrm{N}$-terminal nucleotide-binding domains containing subdomains I-IV, C-terminal phosphotransfer and protein-substrate-binding domains containing subdomains VIa-XI, and the intervening linker containing subdomain $\mathrm{V}[15]$. In addition to the conserved catalytic domains, some STKs contain at least one additional domain, such as FHA, WD40, PAS and GAF [16], which may endow STKs with more complicated functions. For example, the FHA domain can participate in a wide range of processes in bacteria, such as intracellular signal transduction, transcription, protein transport, DNA repair, and protein degradation. Moreover, there is a near-perfect correlation between the presence of FHA-containing proteins and Ser/Thr kinases and phosphatases in bacterial genomes [17].

In cyanobacteria, mutation analyses have revealed the functions of some STKs, such as SpkA and SpkB involved in the cellular motility in Synechocystis PCC6803 [18,19], and PknD involved in the regulation of nitrogen metabolism in Anabaena PCC7120 [20]. A complete signaling pathway involving a bacteria STK remains to be described, but one may anticipate that some STKs are important or even essential in regulating bacterial activities.

As of January 2007, 19 cyanobacterial genomes have been fully sequenced and 6 are in the draft stages. In addition, more than 20 genomes are in the process of being sequenced. The availability of multiple sequenced genomes has been very helpful in phylogenetic and functional studies of cyanobacterial genes, such as those in restriction-modification systems [21] and two-component systems [22]. Comparative genome analysis has been employed in the study on STKs in archaea [15], mycobacteria [23] streptomyces [24] and cyanobacteria [25,26]. Here we present a detailed analysis of the repertoire of STKs in sequenced cyanobacterial genomes. An attempt has been made to identify all STK sequences encoded in the 19 fully sequenced genomes and 2 draft sequences (Crocosphaera watsonii WH8501 and Nostoc punctiforme PCC73102). An analysis of STK sequences according to their classifications, conservation, domain organization, phylogeny and evolution are presented in this paper, aiming towards a deep understanding of the biological role of STKs in cyanobacteria. 


\section{Results Identification of STK proteins}

The 21 cyanobacterial genomes fully available from IMG database (Version 2.0, as of Jan 2007) were considered in our analysis (Fig. 1, Table 1). Synechococcus elongatus PCC6301 and Synechococcus elongatus PCC7942 are virtually identical except for a chromosomal inversion, but both were included in our analysis. Using BlastP and TBlastN programs to look for proteins similar to proven cyanobacterial STKs, we obtained 303 protein sequences from the 21 cyanobacterial genomes, 284 of which were originally annotated as protein kinase or Serine/Threonine Protein Kinase. The remaining 19 proteins were accepted as STKs in this study after sequence alignment and SMART analysis [27]. Seventeen out of these were originally annotated not by their STK domains but by other additional domains, such as ATPase and WD40 repeats, and the last two were annotated as hypothetical proteins [16].

17 proteins, all originally annotated as STKs, from the 303 proteins identified by sequence similarity lack all or part of at least one important catalytic domains [13], and these were excluded from further consideration. Some slightly truncated proteins, such as CwatDRAFT_6607 and CwatDRAFT_1979, lacking structural motifs I, II or XI but possessing all catalytic motifs were retained. Nine proteins (including four STKs in marine unicellular Prochlorococcus and Synechococcus, 6803_slr1443, CwatDRAFT_090 1, Tery_0088, Tery_4781 and Ava_3867) exhibit some deviations from the canonical motifs (Fig. 2) that were deemed tolerable. In all 286 putative STK sequences were considered in this study (Table 1).

The number of STK genes within the different cyanobacterial genomes varies considerably, from 0 to 56. Only one STK gene was found within the 5 fully sequenced Prochlorococcus marinus strains, in strain MIT9313. Eight out of nine sequenced Synechococcus strains contain STKs, with the marine Synechococcus strain WH8102 being the only exception. Three marine Synechococcus strains CC9902, CC9605, and CC9311 contain only one STK gene for each. Two Synechococcus elongatus strains PCC6301 and PCC7942, which are virtually identical in sequence, have five pairs of orthologous STK genes. More STK genes were found in the thermophilic Synechococcus strains JA-3-3Ab (8), JA-2-3Ba (8), and BP-1 (10). The other two unicellular cyanobacterial strains, Gloeobacter violaceus PCC7421

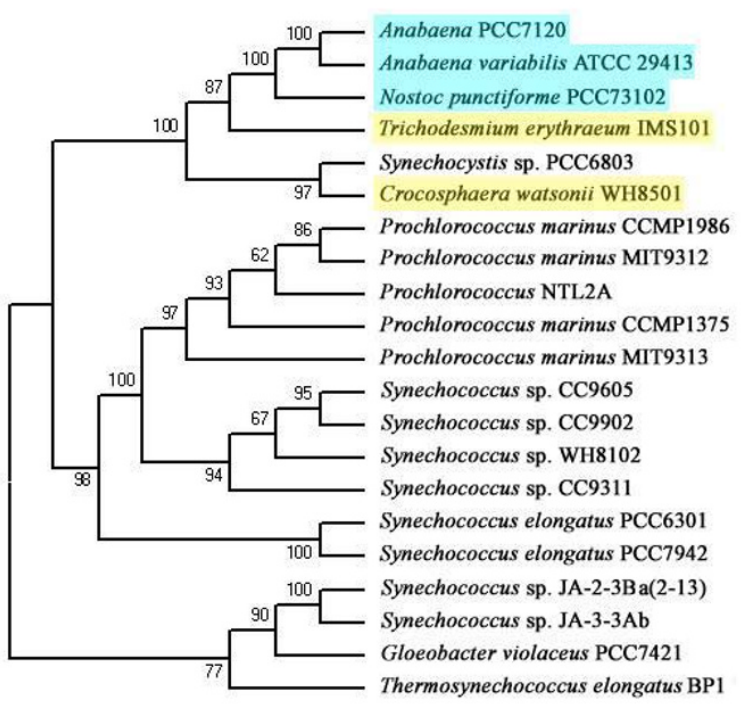

$\begin{array}{lcccc}\text { Key Feature } & \text { Acronym } & \begin{array}{c}\text { Total } \\ \text { Proteins }\end{array} & \begin{array}{c}\text { Total } \\ \text { STKs }\end{array} & \begin{array}{c}\text { Additional } \\ \text { domains }\end{array} \\ \text { Filamentous freshwater } & 7120 & 6214 & 48(0.77 \%) & 27(56.3 \%) \\ \text { Filamentous soil } & \text { Ava } & 5746 & 53(0.92 \%) & 25(47.2 \%) \\ \text { Filamentous symbiont } & \text { Npun } & 7364 & 56(0.76 \%) & 27(48.2 \%) \\ \text { Filamentous marine } & \text { Tery } & 4759 & 40(0.84 \%) & 31(77.5 \%) \\ \text { Unicellular freshwater } & 6803 & 3564 & 7(0.20 \%) & 2(28.6 \%) \\ \text { Unicellular marine } & \text { Cwat } & 5967 & 28(0.47 \%) & 5(17.9 \%) \\ \text { Unicellular marine } & 1986 & 1716 & - & - \\ \text { Unicellular marine } & 9312 & 1808 & - & - \\ \text { Unicellular marine } & \text { NTL2A } & 1894 & - & - \\ \text { Unicellular marine } & 1375 & 1882 & - & - \\ \text { Unicellular marine } & 9313 & 2273 & 1(0.04 \%) & - \\ \text { Unicellular marine } & 9605 & 2695 & 1(0.04 \%) & - \\ \text { Unicellular marine } & 9902 & 2321 & 1(0.04 \%) & - \\ \text { Unicellular marine } & 8102 & 2526 & - & - \\ \text { Unicellular marine } & 9311 & 2892 & 1(0.03 \%) & - \\ \text { Unicellular freshwater } & 6301 & 2527 & 5(0.20 \%) & 1(20.0 \%) \\ \text { Unicellular freshwater } & 7942 & 2662 & 5(0.20 \%) & 1(20.0 \%) \\ \text { Unicellular hot spring } & \text { CYB } & 2862 & 8(0.28 \%) & 2(25.0 \%) \\ \text { Unicellular hot spring } & \text { CYA } & 2760 & 8(0.29 \%) & 3(37.5 \%) \\ \text { Unicellular rock } & 7421 & 4430 & 14(0.32 \%) & 3(21.4 \%) \\ \text { Unicellular hot spring } & \text { TBP1 } & 2475 & 10(0.40 \%) & 4(40.0 \%)\end{array}$

Figure I

Phylogenetic tree of the sequenced cyanobacterial strains and STK information. A phylogenetic tree for 21 sequenced cyanobacteria constructed based on 16s rRNA as was described in Methods. Numbers appearing at the nodes corresponded to the values produced by bootstrap analysis ( 1000 replicates). Names of marine nitrogen-fixing strains are marked in yellow boxes. Filamentous diazotrophic strains capable of heterocyst differentiation are marked in cyan boxes. This tree is similar to that obtained by Ashby and Houmard [22]. Percentages in brackets represent total STKs as a percentage of total proteins and total additional domains as a percentage of total STKs. 
Table I: Cyanobacterial genes encoding Ser/Thr protein kinases

\begin{tabular}{|c|c|c|c|c|c|}
\hline Gene $^{a}$ & Familyb & Additional domains & Gene & Family & Additional domains \\
\hline \multicolumn{6}{|c|}{ Prochlorococcus marinus str. MIT 9313} \\
\hline PMT0I54 & cbSTKI-other & & & & \\
\hline \multicolumn{6}{|c|}{ Synechococcus sp. CC9902 } \\
\hline Syncc9902_0160 & cbSTKI-other & & & & \\
\hline \multicolumn{6}{|c|}{ Synechococcus sp. CC9605 } \\
\hline Syncc9605_0116 & cbSTKI-other & & & & \\
\hline \multicolumn{6}{|c|}{ Synechococcus sp. CC93II } \\
\hline sync_0122 & cbSTKI-other & & & & \\
\hline \multicolumn{6}{|c|}{ Synechococcus elongatus PCC 6301} \\
\hline syc0259_c & cbSTKII & Pentapeptide $_{(2)}$ & syc0757_d & cbSTKI-other & \\
\hline syc0428_d & cbSTKI-other & & syc0923_d & cbSTKI-other & \\
\hline syc0438_d & cbSTKI-other & & & & \\
\hline \multicolumn{6}{|c|}{ Synechococcus elongatus PCC 7942} \\
\hline Synpcc7942_0600 & cbSTKI-other & & Synpcc7942_1121 & cbSTKI-other & \\
\hline Synpcc7942_0780 & cbSTKI-other & & Synpcc7942_1294 & cbSTKII & Pentapeptide $_{(2)}$ \\
\hline Synpcc7942_IIII & cbSTKI-other & & & & \\
\hline \multicolumn{6}{|c|}{ Synechocystis sp. PCC6803 } \\
\hline sll I574(SpkA) & cbSTKI-other & & slr0I52(SpkG) & cbSTKI-other & \\
\hline slr I225(SpkF) & cbSTKI-TM & & sll0776(SpkD) & cbSTKII & $\mathrm{SH} 3 \mathrm{~b}$ \\
\hline slr I443(SpkE) & cbSTKI-other & & slr0599(SpkC) & cbSTKI-other & \\
\hline slr I697(SpkB) & cbSTKII & Pentapeptide $_{(2)}$ & & & \\
\hline \multicolumn{6}{|c|}{ Synechococcus sp. JA-2-3Ba(2-13) } \\
\hline CYB_0466 & cbSTKI-other & & CYB_I522 & cbSTKII & Pentapeptide \\
\hline CYB_0522 & cbSTKI-other & & CYB_I864 & cbSTKI-other & \\
\hline CYB_0637 & cbSTKII & GUN4 & CYB_25I5 & cbSTKI-other & \\
\hline CYB_0949 & cbSTKI-other & & CYB_2575 & cbSTKI-other & \\
\hline \multicolumn{6}{|c|}{ Synechococcus sp. JA-3-3Ab } \\
\hline CYA_0209 & cbSTKI-other & & CYA_2262 & cbSTKI-other & \\
\hline CYA_0373 & cbSTKI-other & & CYA_2333 & cbSTKII & $\operatorname{TPR}_{(18)}$ \\
\hline CYA_1882 & cbSTKII & Pentapeptide & CYA_2756 & cbSTKII & GUN4 \\
\hline CYA_2I46 & cbSTKI-other & & CYA_2859 & cbSTKI-other & \\
\hline \multicolumn{6}{|c|}{ Thermosynechococcus elongatus BPI } \\
\hline tlr0445 & cbSTKI-other & & $\operatorname{t|r} \mid 460$ & cbSTKI-other & \\
\hline $\operatorname{t}|r| 098$ & cbSTKI-other & & $\operatorname{tr} 1540$ & cbSTKII & CHASE2 \\
\hline $\operatorname{tr} \mid 128$ & cbSTKII & FHA & tll2222 & cbSTKI-other & \\
\hline tlll 205 & cbSTKII & CHASE2 & $\operatorname{t} \operatorname{lr} 2304$ & cbSTKI-other & \\
\hline $\operatorname{t}|r| 326$ & cbSTKII & Pentapeptide $_{(2)}$ & $\operatorname{tr} 2432$ & cbSTKI-TM & \\
\hline \multicolumn{6}{|c|}{ Gloeobacter violaceus PCC 7421} \\
\hline gll0054 & cbSTKI & & glr 1346 & cbSTKI-other & \\
\hline glr0422 & cbSTKI-other & & glr| 552 & cbSTKI-other & \\
\hline gll0585 & cbSTKII & $\mathrm{TPR}_{(3)}$ & g112103 & cbSTKI & \\
\hline glr0657 & cbSTKI-other & & gl|2127 & cbSTKI & \\
\hline glr0665 & cbSTKI & & glr4017 & cbSTKI & \\
\hline glr09I5 & cbSTKI-other & & glr4072 & cbSTKII & $\operatorname{TPR}_{(5)}$ \\
\hline $\operatorname{glr} 1096$ & cbSTKII & $\operatorname{TPR}_{(4)}$ & glr4| 07 & cbSTKI & \\
\hline \multicolumn{6}{|c|}{ Crocosphaera watsonii WH850I } \\
\hline CwatDRAFT_0326 & cbSTKI-other & & CwatDRAFT_3230 & cbSTKII & Pentapeptide $_{(3)}$ \\
\hline CwatDRAFT_0327 & cbSTKI & & CwatDRAFT_3247 & cbSTKI & \\
\hline CwatDRAFT_0457 & cbSTKI & & CwatDRAFT_3998 & cbSTKII & GUN4 \\
\hline CwatDRAFT_090I & cbSTKI & & CwatDRAFT_4660 & cbSTKI-other & \\
\hline CwatDRAFT_0902 & cbSTKII & ANF & CwatDRAFT_4756 & cbSTKI-other & \\
\hline CwatDRAFT_I649 & cbSTKII & $\mathrm{PbHI}_{(3)}$ & CwatDRAFT_476I & cbSTKI-other & \\
\hline CwatDRAFT_I757 & cbSTKI-other & & CwatDRAFT_4762 & cbSTKI-other & \\
\hline CwatDRAFT_I955 & cbSTKI-other & & CwatDRAFT_4879 & cbSTKII & $\mathrm{WD} 40_{(7)}$ \\
\hline CwatDRAFT_I979 & cbSTKI & & CwatDRAFT_4783 & cbSTKI-other & \\
\hline CwatDRAFT_2418 & cbSTKI & & CwatDRAFT_5890 & cbSTKI-other & \\
\hline CwatDRAFT_2794 & cbSTKI-other & & CwatDRAFT_6040 & cbSTKI & \\
\hline CwatDRAFT_2895 & cbSTKI-other & & CwatDRAFT_6269 & cbSTKI & \\
\hline CwatDRAFT_3022 & cbSTKI & & CwatDRAFT_6420 & cbSTKI & \\
\hline
\end{tabular}


Table I: Cyanobacterial genes encoding Ser/Thr protein kinases (Continued)

\begin{tabular}{|c|c|c|c|c|c|}
\hline \multirow{2}{*}{\multicolumn{6}{|c|}{$\begin{array}{l}\text { CwatDRAFT_3I84 cbSTKI-TM } \\
\text { Trichodesmium erythraeum IMS I } 0 \text { I }\end{array}$}} \\
\hline \multirow{2}{*}{\multicolumn{6}{|c|}{$\begin{array}{l}\text { Trichodesmium erythraeum IMS I 0 I } \\
\begin{array}{lll}\text { Tery } 0059 & \text { cbSTKII } & \text { WD } 40\end{array}\end{array}$}} \\
\hline & & & & & \\
\hline Tery_0088 & cbSTKII & $\mathrm{FHA}$ & Tery_2556 & cbSTKIII & GAF, HiskA \\
\hline Tery_0184 & cbSTKII & $\mathrm{WD} 40_{(7)}$ & Tery_2609 & cbSTKII & RDD \\
\hline Tery_0247 & cbSTKII & DUF323 & Tery_2829 & cbSTKII & DUF323 \\
\hline Tery_0460 & cbSTKII & DUF323 & Tery_283I & cbSTKII & DUF323 \\
\hline Tery_046I & cbSTKII & DUF323 & Tery_2857 & cbSTKII & GAF, CYCc \\
\hline Tery_0462 & cbSTKII & DUF323 & Tery_3150 & cbSTKI & \\
\hline Tery_0463 & cbSTKII & DUF323 & Tery_3337 & cbSTKII & $\mathrm{TPR}_{(3)}$ \\
\hline Tery_062I & cbSTKII & FHA & Tery_3345 & cbSTKI-other & \\
\hline Tery_0858 & cbSTKII & $\operatorname{TPR}_{(3)}$ & Tery_3400 & cbSTKII & Pentapeptide $_{(5)}$ \\
\hline Tery_1030 & cbSTKII & $\mathrm{FHA}$ & Tery_3423 & cbSTKI-other & \\
\hline Tery_1036 & cbSTKII & DUF323 & Tery_3510 & cbSTKI & \\
\hline Tery_1627 & cbSTKII & WD40 $_{(7)}$ & Tery_368I & cbSTKII & $\operatorname{TPR}_{(7)}$ \\
\hline Tery_1668 & cbSTKI-other & & Tery_3863 & cbSTKII & $\mathrm{GAF}$ \\
\hline Tery_I722 & cbSTKII & GUN4 & Tery_4060 & cbSTKII & $\mathrm{WD} 40_{(7)}$ \\
\hline Tery_I723 & cbSTKII & GUN4 & Tery_435I & cbSTKII & DUF323 \\
\hline Tery_2033 & cbSTKII & $\mathrm{WD} 40_{(7)}$ & Tery_44II & cbSTKI-TM & \\
\hline Tery_205I & cbSTKI-other & & Tery_4467 & cbSTKII & WD40 $_{(7)}$ \\
\hline Tery_2064 & cbSTKII & Pentapeptide $_{(3)}$ & Tery_478I & cbSTKII & $\operatorname{TPR}_{(I I)}$ \\
\hline Tery_2107 & cbSTKI-other & & Tery_4782 & cbSTKII & $\operatorname{TPR}_{(10)}$ \\
\hline \multicolumn{6}{|c|}{ Anabaena PCC7I 20} \\
\hline all0192 & cbSTKI-other & & alr3119 & cbSTKII & $\mathrm{WD}{ }_{(7)}$ \\
\hline all0323 & cbSTKIII & GAF, HisKA & all3169 & cbSTKII & WD40 \\
\hline alr0344 & cbSTKII & ANF & all3206 & cbSTKI-other & \\
\hline alr0354 & cbSTKIII & GAF, HisKA & all3207 & cbSTKI-other & \\
\hline all0438 & cbSTKII & $\mathrm{WD} 40_{(7)}$ & alr3268 & cbSTKII & Pentapeptide $_{(2)}$ \\
\hline alr0548 & cbSTKII & FHA & all3557 & cbSTKIII & GAF, HisKA \\
\hline alr0709 & cbSTKIII & GAF, HisKA & all369l & cbSTKIII & GAFGAF, HisKA \\
\hline alr07I0 & cbSTKIII & GAF, HisKA & alr3706 & cbSTKI-TM & \\
\hline all0886 & cbSTKIII & GAF, HisKA & alr3732(PknE) & cbSTKI-other & \\
\hline alr0900 & cbSTKIII & GAF, HisKA & all3773 & cbSTKII & $\operatorname{TPR}_{(10)}$ \\
\hline $\operatorname{alr}|3| \mid$ & cbSTKI & & alr3877 & cbSTKI-other & \\
\hline alr 1336 & cbSTKII & DUF323 & alr3997 & cbSTKI-other & \\
\hline all 1625 & cbSTKIII & GAF, HisKA & alr4I4I & cbSTKI & \\
\hline alr 1869 & cbSTKII & CHASE2 & alr4366(PknA) & cbSTKI-other & \\
\hline all1919 & cbSTKI-other & & alr4368(PknD) & cbSTKI-other & \\
\hline alr2258 & cbSTKIII & GAF, HisKA & all4518 & cbSTKI-other & \\
\hline alr2259(PknB) & cbSTKI-other & & all4668 & cbSTKI-other & \\
\hline all2282 & cbSTKIII & GAF, HisKA & all4687 & cbSTKIII & GAF, HisKA \\
\hline all2334 & cbSTKI-TM & & all48I3(PknC) & cbSTKI-other & \\
\hline alr24II & cbSTKI-other & & all4838 & cbSTKII & CHASE2 \\
\hline alr2412 & cbSTKI-other & & alr4949 & cbSTKII & GUN4 \\
\hline alr2502 & cbSTKII & $\mathrm{PbHI}_{(8)}$ & alr4954 & cbSTKII & FHA \\
\hline alr2682 & cbSTKIII & GAF, PAS, HisKA & all5278 & cbSTKI & \\
\hline all2760 & cbSTKI & & alr7232 & cbSTKII & $\operatorname{TPR}_{(3)}$ \\
\hline \multicolumn{6}{|c|}{ Anabaena variabilis ATCC 29413} \\
\hline Ava_00I2 & cbSTKI & & Ava_285I & cbSTKII & WD40 ${ }_{(6)}$ \\
\hline Ava_0083 & cbSTKIII & GAF, HiskA & Ava_2989 & cbSTKI & \\
\hline Ava_0084 & cbSTKI-other & & Ava_2990 & cbSTKI-TM & \\
\hline Ava_0I53 & cbSTKI-TM & & Ava_3036 & cbSTKI & \\
\hline Ava_0219 & cbSTKI-other & & Ava_3308 & cbSTKI-other & \\
\hline Ava_0220 & cbSTKI-other & & Ava_3310 & cbSTKI-other & \\
\hline Ava_0353 & cbSTKII & $\mathrm{FHA}$ & Ava_3384 & cbSTKI-other & \\
\hline Ava_0434 & cbSTKII & $\mathrm{PbHI}_{(8)}$ & Ava_3535 & cbSTKIII & GAF, HiskA \\
\hline Ava_0762 & cbSTKI & & Ava_3584 & cbSTKI-TM & \\
\hline Ava_II53 & cbSTKI & & Ava_3596 & cbSTKIII & GAFGAF, HiskA \\
\hline Ava_I552 & cbSTKII & $\operatorname{TPR}_{(10)}$ & Ava_3867 & cbSTKII & $\mathrm{WD}^{4} 0_{(7)}$ \\
\hline Ava_I592 & cbSTKI-other & & Ava_3995 & cbSTKIII & GAF, HiskA \\
\hline Ava_I 1700 & cbSTKI-other & & Ava_4l06 & cbSTKI-other & \\
\hline Ava_1816 & cbSTKI-other & & Ava_439I & cbSTKI-other & \\
\hline Ava_1980 & cbSTKIII & GAF, HiskA & Ava_4489 & cbSTKIII & GAF, HiskA \\
\hline
\end{tabular}


Table I: Cyanobacterial genes encoding Ser/Thr protein kinases (Continued)

\begin{tabular}{|c|c|c|c|c|c|}
\hline Ava_1995 & cbSTKI-other & & Ava_4503 & cbSTKIII & GAF, HiskA \\
\hline Ava_2084 & cbSTKI-other & & Ava_4504 & cbSTKIII & GAF, HiskA \\
\hline Ava_2109 & cbSTKII & CHASE2 & Ava_4716 & cbSTKIII & GAF, HiskA \\
\hline Ava_2519 & cbSTKI-other & & Ava_4764 & cbSTKII & CHASE2 \\
\hline Ava_2528 & cbSTKI & & Ava_4855 & cbSTKII & WD40 $_{(7)}$ \\
\hline Ava_2684 & cbSTKI-other & & Ava_4862 & cbSTKI-other & \\
\hline Ava_279I & cbSTKII & ANF & Ava_4863 & cbSTKI-other & \\
\hline Ava_2803 & cbSTKIII & GAF, HiskA & Ava_4923 & cbSTKII & Pentapeptide $_{(2)}$ \\
\hline Ava_2809 & cbSTKI-other & & Ava_5054 & cbSTKII & DUF323 \\
\hline Ava_2845 & cbSTKIII & GAF, PAC, HiskA & Ava_A0032 & cbSTKII & $\operatorname{TPR}_{(6)}$ \\
\hline Ava_B0009 & cbSTKII & TPR & Ava_B0202 & cbSTKI-other & \\
\hline Ava_COII7 & cbSTKIII & GAF(PAS, PAC)4PAS HisKA & & & \\
\hline \multicolumn{6}{|c|}{ Nostoc punctiforme PCC73 102} \\
\hline Npun02000I03 & cbSTKIII & GAF, HiskA & Npun02004756 & cbSTKIII & GAFPASGAF, HiskA \\
\hline Npun02000I63 & cbSTKI-other & & Npun02004875 & cbSTKII & CHASE2 \\
\hline Npun02000400 & cbSTKII & $\mathrm{WD} 40_{(7)}$ & Npun02005090 & cbSTKI & \\
\hline Npun02000527 & cbSTKI & & Npun02005I7I & cbSTKII & $\mathrm{WD} 40_{(7)}$ \\
\hline Npun0200094I & cbSTKIII & GAF, PAS, PAC, HiskA & Npun02005475 & cbSTKI & \\
\hline Npun0200II34 & cbSTKIII & GAF, HiskA & Npun02005477 & cbSTKII & WD40 $_{(7)}$ \\
\hline Npun0200II 48 & cbSTKIII & GAF, HiskA & Npun0200569I & cbSTKI-other & \\
\hline Npun02001995 & cbSTKI-other & & Npun02006I73 & cbSTKIII & GAF, HiskA \\
\hline Npun02002I75 & cbSTKI & & Npun02006290 & cbSTKIII & GAF, HiskA \\
\hline Npun02002176 & cbSTKI-other & & Npun02006325 & cbSTKI-TM & \\
\hline Npun0200230I & cbSTKI-other & & Npun02006559 & cbSTKI-other & \\
\hline Npun020024I8 & cbSTKI-other & & Npun02006688 & cbSTKI-other & \\
\hline Npun0200242I & cbSTKII & $\operatorname{TPR}_{(10)}$ & Npun02006812 & cbSTKI-TM & \\
\hline Npun02002449 & cbSTKI-other & & Npun020068I3 & cbSTKI & \\
\hline Npun02002654 & cbSTKII & $\operatorname{TPR}_{(10)}$ & Npun02006829 & cbSTKI-other & \\
\hline Npun02002842 & cbSTKI-other & & Npun02006849 & cbSTKI-other & \\
\hline Npun02002843 & cbSTKI-other & & Npun02007024 & cbSTKII & WD40 $_{(7)}$ \\
\hline Npun02003 II4 & cbSTKI-other & & Npun02007058 & cbSTKI-other & \\
\hline Npun02003I77 & cbSTKII & $\operatorname{TPR}_{(3)}$ & Npun02007062 & cbSTKIII & GAF, HiskA \\
\hline Npun02003225 & cbSTKI-other & & Npun02007070 & cbSTKII & DUF323 \\
\hline Npun02003227 & cbSTKII & WD40 $_{(7)}$ & Npun02007I95 & cbSTKII & CHASE2 \\
\hline Npun02003383 & cbSTKI-other & & Npun02007679 & cbSTKIII & GAF, HiskA \\
\hline Npun0200357I & cbSTKII & CHASE2 & Npun02007917 & cbSTKI-TM & \\
\hline Npun02003917 & cbSTKIII & GAF(PASPAC)2 HiskA & Npun02007926 & cbSTKI-TM & \\
\hline Npun02004022 & cbSTKII & $\mathrm{TPR}_{(4)}$ & Npun02008046 & cbSTKI-other & \\
\hline Npun02004075 & cbSTKII & $\mathrm{FHA}$ & Npun02008I59 & cbSTKIII & GAF, HiskA \\
\hline Npun02004457 & cbSTKIII & $\mathrm{GAF}_{(3)}$, PAS, PAC, HiskA & Npun02008276 & cbSTKI-other & \\
\hline Npun02004619 & cbSTKI & & Npun02008678 & cbSTKII & Pentapeptide $_{(2)}$ \\
\hline
\end{tabular}

and Crocosphaera watsonii WH8501, have 14 and 28 STK genes respectively.

Filamentous diazotrophic cyanobacteria have the largest number of STK genes (40 for Trichodesmium erythraeum IMS101, 48 for Anabaena PCC7120, 53 for Anabaena variabilis ATCC 29413, and 56 for Nostoc punctiforme PCC73102). Among the unicellular cyanobacteria, the nitrogen-fixing unicellular strain Crocosphaera WH8501 has the biggest genome, the largest number of STKs and the highest percentage $(0.52 \%)$ of STKs within proteinencoding genes (Fig. 1). STKs in Prochlorococcus MIT9313 represent only $0.04 \%$ of the total proteins, while in Anabaena ATCC29413, the percentage is twenty-fold higher at
$0.85 \%$. It is evident from these findings that filamentous diazotrophic cyanobacteria contain more STK genes than unicellular species (T-test, $p<0.001$ ), and the number of STK genes is overrepresented in their genomes even after allowing for their larger genome sizes (Fig. 1).

\section{Conserved domain features}

The core catalytic domain contains 12 conserved subdomains, within which a total of 12 amino acids are invariant or nearly invariant in eukaryotes: two $G$ residues in subdomain I, K in subdomain II, E in subdomain III, D and $\mathrm{N}$ in subdomain VIb, D and G in subdomain VII, E in subdomain VIII, D and G in subdomain IX, and R in subdomain XI [13]. In cyanobacteria, 12 similar conserved 

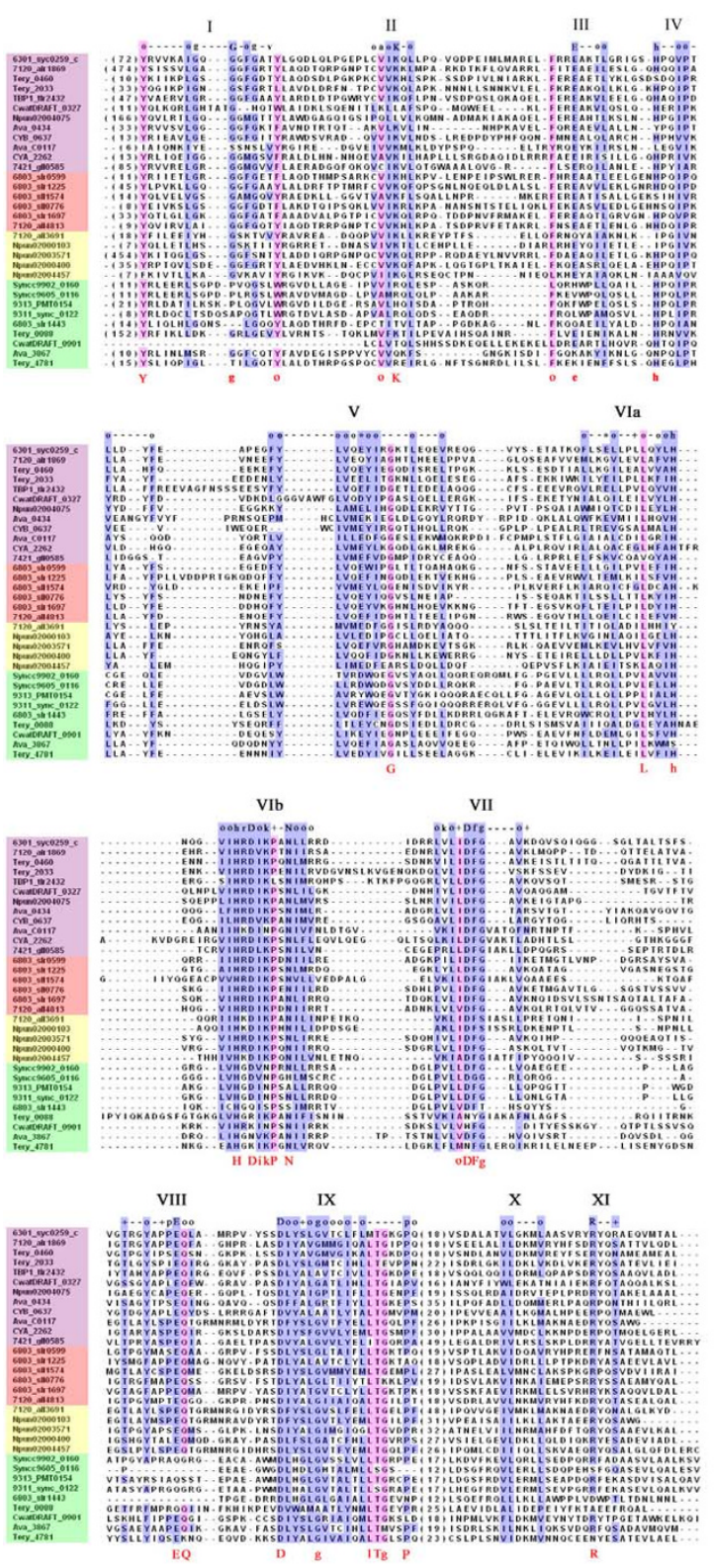

\section{Figure 2}

Conserved domains and sites in cyanobacterial STKs according to Hanks and Hunter. A total of 32 sequences were used in the alignment of cyanobacterial STKs: 12 sequences in purple were selected to cover the diversity of species and structural characteristics; 6 sequences in red are of proteins reported to possess phosphorylation or autophosphorylation activity; 5 sequences in yellow were not originally annotated as protein kinases or Serine/Threonine Protein Kinases; and 9 sequences in green were those showing some deviations from the canonical domains of Hanks and Hunter [13]. Each sequence is denoted by the species name followed by the gene names. The 12 conserved subdomains are indicated by Roman numerals according to Hanks and Hunter. Highly conserved eukaryotic and cyanobacteria-specific amino-acid residues are indicated above and below the alignment sequences respectively, using the single-letter amino-acid classes of Hanks and Hunter. Symbols, used to denote the various features according to Hanks and Hunter, are as follows: uppercase letters, universally conserved amino acid residues; lowercase letters, highly conserved amino acid residues; o, positions conserving non-polar residues; *, positions conserving polar residues; +, positions conserving small residues with near neutral polarity; and -, positions within a subdomain with no pattern of conserved amino acids. The number in parentheses preceding each sequence refers to the number of amino acid residues preceeding the sequence shown. 
subdomains were found and 10 residues are conserved well, with the exception of the two G residues in subdomain I, which are common but not invariant. It should be noted that additional conserved amino acids appear in cyanobacterial subdomains I, III, V, VIa, VIb, VII, VIII and IX (Fig. 2).

In eukaryotes, subdomain I serves as the ATP binding site (P-loop) and is characterized by the sequence GXGXXG. Cyanobacterial STKs are divergent in this motif with only $152(53.3 \%)$ sequences exactly conserved in this domain, but $94.4 \%$ of them contain at least one Gly. It is possible that STKs lacking a conventional subdomain I may employ high energy phosphate compounds other than ATP, as does a PK from Sulfolobus acidocaldarius that was reported to use polyphosphate as phosphoryl donor $[28,29]$. Besides the conserved Gly, cyanobacterial subdomain I differs from its eukaryotic counterpart in that it starts at Tyr (93\% of all cyanobacterial sequences) and ends with a non-polar residues (99\%).

The Lys $(96.1 \%)$ in subdomain II is important for maximal catalytic activity and helping anchor and orient ATP, and it is well conserved in cyanobacteria. But the upstream Ala described by Hanks and Hunter [13] is generally replaced by a different non-polar residue $(97.2 \%)$. In subdomain III, Glu (86.0\%) is not conserved well and is substituted by Gln in 27 sequences belonging to cyanobacterial Family III (cbSTKIII, details in section Structure and function). Upstream of the Glu, a conserved nonpolar residue $(99.3 \%)$ was found and most of them were Phe $(87.7 \%)$.

Cyanobacterial subdomain $\mathrm{V}$ is much more conserved than the eukaryotic counterpart, which has no invariant residues. In contrast, the cyanobacterial subdomain possesses a highly conserved Gly (94.1\%). This conserved residue may help anchor ATP by forming hydrogen bonds. Gly is also conserved in other bacteria (e.g. Streptomyces coelicolor [24]). In eukaryotes, subdomain VIa contributes to structural stabilization and possesses only a relatively conserved His. In cyanobacteria, besides this His (88.1\%), a more conserved Leu (97.2\%) has been found, which is also conserved well in Mycobacterium tuberculosis [23].

Subdomain VIb, a candidate for direct catalysis, is the most conserved motif in eukaryotes and is characterized as "hrDxkxxN". Cyanobacterial subdomain VIb is more conserved and is characterized as "HrDikPxN". His $(100 \%)$ is the most conserved residue, followed by Asn (98.9\%), Pro (98.6\%), Asp (97.2\%), Lys (86.7\%), Ile $(82.5 \%)$ and $\operatorname{Arg}(73.8 \%)$. Pro is also conserved in other bacteria, such as Streptomyces coelicolor [24] and Mycobacterium tuberculosis [23]. STKs are also referred as "RD" kinases because their activation requires the Arg and cata- lytic Asp in this subdomain. In cyanobacteria, however, Arg is not conserved well and it can be substituted by Lys, Gly, Cys or Gln. All members of cyanobacterial Family III (cbSTKIII) belong to the family of "KD" kinases. There are five cyanobacterial STKs lacking the highly conserved D, a candidate for the catalytic base. Among these five putative STKs, SpkE (Slr1443), which also lack of several other conserved residues, did not show any kinase activity [30]. Whether the other four could phosphorylate or not may need more experimental proofs.

Subdomain VII helps to orient the $\gamma$ phosphate of ATP for transfer and is characterized by a conserved DFG triplet. The invariant Asp in subdomain VII, as well as the invariant Lys in subdomain II, was found to anchor and orient ATP [13]. In cyanobacteria, Asp (97.2\%) and Phe (97.9\%) are highly conserved but Gly (87.4\%) less so. In 30 STK proteins from cyanobacterial Family III (cbSTKIII), Gly is substituted by Ser. The Lys residue often found in eukaryotes before the DFG triplet is not conserved in cyanobacteria, and the residues with near neutral polarity were replaced by non-polar ones (95.1\%).

The typical subdomain VIII includes a highly conserved PE motif and plays a major role in the recognition of peptide or protein substrates. In cyanobacteria, the Pro residue $(62.2 \%)$ of this motif is not well conserved, and the Glu residue $(97.5 \%)$ is followed by a conserved Gln residue $(90.5 \%)$ of unknown function. The PE motif in archaea, eubacteria and eukaryotes may represent an ancient and universal substrate recognition mechanism.

In subdomain IX, besides the Asp (99.3\%), Gly (84.6\%) and Pro (90.2\%) residues conserved in eukaryotes, another three conserved amino acids were found: Thr (90.9\%), Leu (87.0\%) and Gly (80.1\%). Gly is also conserved in Mycobacterium tuberculosis [23] and Streptomyces coelicolor [24].

In cyanobacteria there are six STKs, SpkA (Sll1574) [18], SpkB (Slr1697) [19], SpkC (Slr0599), SpkD (Sll0776), SpkF (Slr1225) [30], PknC (All4813) [31], which have been shown to phosphorylate themselves or other substrate proteins. From the sequence alignment it can seen that all six of these STKs have sequences that are in accordance with the conserved features found in cyanobacteria (Fig. 2).

\section{Structure and function}

Cyanobacterial STKs were classified according to structural characteristics into three major families: cbSTKI, cbSTKII and cbSTKIII (Fig. 3).

Cyanobacterial STK Family I (cbSTKI) groups together the $155(54.2 \%)$ STK proteins that possess no identifiable 


\begin{tabular}{|c|c|c|c|c|c|c|c|c|c|c|c|c|c|c|c|c|c|}
\hline \multirow{2}{*}{ Families } & \multirow{2}{*}{ Architecture } & \multicolumn{16}{|c|}{ Distribution } \\
\hline & & 9313 & 9902 & 9605 & 9311 & 6301 & 7942 & CYB & CYA & TBP1 & 7421 & 6803 & Cwat & Tery & 7120 & Ava & Npun \\
\hline \multirow{3}{*}{ cbSTKI } & \multirow{3}{*}{ STK } & & & & & & & & & & 6 & & 11 & 2 & 4 & 6 & 6 \\
\hline & & & & & & & & & & 1 & & 1 & 1 & 1 & 2 & 3 & 4 \\
\hline & & 1 & 1 & 1 & 1 & 4 & 4 & 6 & 5 & 5 & 5 & 4 & 11 & 6 & 15 & 19 & 19 \\
\hline \multirow{14}{*}{ cbSTKII } & \multirow{14}{*}{ 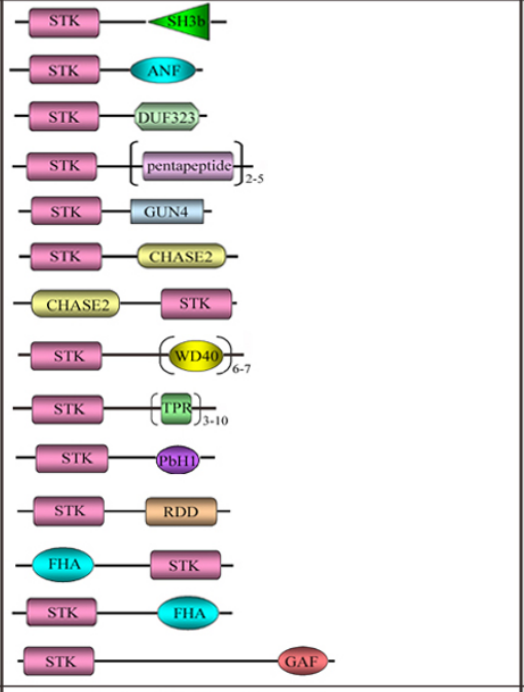 } & & & & & & & & & & & 1 & & & & & \\
\hline & & & & & & & & & & & & & 1 & & 1 & 1 & \\
\hline & & & & & & & & & & & & & & 9 & 1 & 1 & 1 \\
\hline & & & & & & 1 & 1 & 1 & 1 & 1 & & 1 & 1 & 2 & 1 & 1 & 1 \\
\hline & & & & & & & & 1 & 1 & & & & 1 & 2 & 1 & & \\
\hline & & & & & & & & & & & & & & & & & 1 \\
\hline & & & & & & & & & & 2 & & & & & 2 & 2 & 2 \\
\hline & & & & & & & & & & & & & 1 & 6 & 3 & 3 & 5 \\
\hline & & & & & & & & & 1 & & 3 & & & 5 & 2 & 3 & 4 \\
\hline & & & & & & & & & & & & & 1 & & 1 & 1 & \\
\hline & & & & & & & & & & & & & & 1 & & & \\
\hline & & & & & & & & & & 1 & & & & 2 & 2 & 1 & 1 \\
\hline & & & & & & & & & & & & & & 1 & & & \\
\hline & & & & & & & & & & & & & & 2 & & & \\
\hline \multirow{9}{*}{ cbSTKIII } & - STK $-/ /$ - GAP HiskA- & & & & & & & & & & & & & 1 & 11 & 9 & 8 \\
\hline & STK $-1 /$ GAE & & & & & & & & & & & & & & 1 & 1 & \\
\hline & STK - $/ /$ - GAPP PAS & & & & & & & & & & & & & & 1 & & \\
\hline & - STK - $/ 1$-GAP-PAC-HiskA- $\triangle T$ TIPase- & & & & & & & & & & & & & & & 1 & \\
\hline & - STK - I/ GAF PAS PAC HisKA ATPase- & & & & & & & & & & & & & & & & 1 \\
\hline & 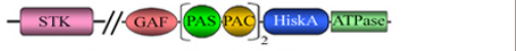 & & & & & & & & & & & & & & & & 1 \\
\hline & AC) PAS HiskA ATPase- & & & & & & & & & & & & & & & 1 & \\
\hline & 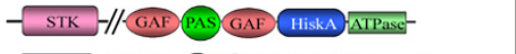 & & & & & & & & & & & & & & & & 1 \\
\hline & STK $-1 /$ & & & & & & & & & & & & & & & & 1 \\
\hline
\end{tabular}

\section{Figure 3}

Schematic representation and distribution of putative cyanobacterial serine/threonine protein kinases. Strain names are as in Figure I.

domain besides that associated with core catalysis. This family was further divided into three subfamilies. Subfamily I (cbSTKI-I) contains 35 proteins with fewer than 400 amino acids long and may be considered to possess the basic structure supplemented in other families by additional domains. Proteins in this family are found only in Gloeobacter PCC7421, Crocosphaera WH8501 and the four filamentous diazotrophic strains. Subfamily II (cbSTKI-TM) contains 13 proteins and is named for their 3 to 6 transmemberane (TM) domains behind the STK domains. These 13 proteins are distributed amongst unicellular Synechocystis PCC6803, Thermosynechococcus elongatus BP1, Crocosphaera watsonii WH 8501 and the four filamentous diazotrophic strains. Subfamily III (cbSTKI- other) consists of 107 STK proteins distributed amongst all 16 strains, each protein with more than 400 amino acids and possessing a long unidentified C-terminal domains. The functions of two cbSTKI proteins in Synechocystis PCC6803 and three in Anabaena PCC7120 have been confirmed by experiment. In Synechocystis PCC6803, Sll1574 (SpkA, I-other) was found to be involved in regulating cellular motility via phosphorylation of membrane proteins [18]. No phenotype defect could be discerned in a knockout mutant of Slr0599 (SpkC, I-other); however the protein was demonstrated to phosphorylate itself and some general substrate proteins [30]. In Anabaena PCC7120, Alr4366 (PknA, I-other) showed a complex pattern of regulation during heterocyst development [32], 
and Alr4368 (PknD, I-other) appeared essential for normal growth under diazotrophic conditions [20]. The function of Alr3732 (PknE, I-other) is known to be required for the formation of heterocyst envelope structures and nitrogen fixation [33].

Cyanobacterial STK Family II (cbSTKII) includes 92 (32.2\%) proteins from 12 cyanobacterial species and are defined as those STK proteins that possess at least one additional domain in addition to their STK domains but do not possess Hik kinase domains. The additional domains with distinct functions are prevalent in protein kinases and phosphatases of prokaryotic and eukaryotic signal transduction systems. In total, 14 types of additional functional domains were identified in cyanobacterial STKs: PAS, PAC, GAF, ANF, WD40, FHA, GUN4, TPR, DUF323, PbH1, CHASE2, Pentapeptide, RDD, and SH3b. Most are involved in known signaling proteins and play various functions in the signal transduction process. For example, TPR (tetratricopeptide repeat), which are repeated in chaperone, cell-cycle, transcription, and protein transport complexes, form signaling domains in higher eukaryotes and may participate in the aggregation of proteins into multi-protein complexes [34]. GAF domains (cyclic GMP, adenylyl cyclases, FhlA) are linked to the binding of small-molecules, in particular the second messengers cyclic AMP (cAMP) and cGMP [35]. WD40 repeats, mainly in eukaryotic proteins, cover a wide variety of functions, for example, in adaptor/regulatory modules in signal transduction $[36,37]$. Finally, GUN4 is thought to participate in plastid-to-nucleus signaling by regulating magnesium-protoporphyrin IX synthesis or trafficking [38]. There are also some domains with unknown functions. These are found mainly in bacteria and include DUF323, Pentapeptide, and RDD. The putative functions of most of these additional domains have been described in detail by Krupa [25]. Most of these additional domains are located at C-terminal, with the exceptions of FHA and CHASE2.

Unicellular non-nitrogen-fixing strains have none or a limited number of additional domains. TPR is identified exclusively in Gloeobacter violaceus PCC7421, and DUF323 is the most prevalent in Trichodesmium erythraeum IMS101. Crocosphaera watsonii WH8501 has the lowest percent of additional domains except for marine unicellular non-nitrogen-fixing strains (Fig. 1). Filamentous diazotrophic cyanobacteria not only contain vast number of STKs but their STKs also recruit a diversity of additional domains. The STKs of Anabaena PCC7120, for example, contain 12 types of different additional domains. The marine filamentous diazotrophic strain Trichodesmium shows an exceptionally high percent (77.5\%) and the greatest number of additional domains. Pentapeptide repeats, which have been found to participate in the accu- mulation of glycolipids into the heterocysts [39], are prevalent in both unicellular and filamentous cyanobacteria. WD40, containing 6 or 7 repeats, are found exclusively in nitrogen-fixing strains. Functions of three proteins in this family have been identified. Alr2502 (Pkn22, PbH1) was found to be associated with both iron-depletion and oxidative stress in Anabaena PCC7120 [40]. In Synechocystis PCC6803, Slr1697 (SpkB, Pentapeptide) was shown to be involved in the control of cell motility exclusive of positive phototaxis [19], and Sll0776 (SpkD, SH3b) could not be knocked out completely, indicating that it is essential for survival [30].

Kinases of STK Family III (cbSTKIII) are also named as dual protein kinases, as they contain both N-terminal STK domains and C-terminal His kinase domains, with at least one GAF domain in between. They are encountered in quite large numbers (38) in filamentous nitrogen-fixing strains, with the exception of Trichodesmium erythraeum IMS101, which possesses only one. This observation is consistent with an earlier comparative study reporting that GAF and PAS domains, possibly involved in signal recognition, are extremely abundant in Anabaena: $87 \mathrm{GAF}$ domains in 62 ORFs and 140 PAS domains in 59 ORFs [41]. The expression of one cbSTKIII protein, Alr2258 (HstK) from Anabaena PCC7120, has been shown to depend on the type of available nitrogen source [42], and the expression of two others, Alr0709 (Pkn40) and Alr0710 (Pkn41), whose genes are adjacent along the chromosome and co-transcribed, are induced by iron deprivation and are under the control of the global nitrogenregulator NtcA [43].

\section{Phylogenetic analysis}

Phylogenetic analysis was performed using the conserved catalytic domains of STKs rather than their whole sequences, as the additional domains with their possibly separate evolutionary histories might confuse the phylogeny. The STK catalytic domains, about 280 amino acids in length, were identified using SMART and CDD databases $[27,44]$.

The STK phylogenetic tree was rooted in the archaeal STKs, deviating from the canonical view of the universal phylogenetic tree rooted in eubacteria [45]. This STK phylogenetic tree indicates that these STK proteins display complicated relationships, at odds with the phylogeny of the species. STK proteins from the unicellular marine Synechococcus and Prochlorococcus cluster together and have a close relationship with Slr1443 from Synechocystis PCC6803. STKs from the unicellular cyanobacteria, Synechocystis PCC6803, Synechococcus elongatus PCC6301, Synechococcus elongatus PCC 7942, and Thermosynechococcus elongatus BP1 are dispersed throughout the phylogenetic tree. Seven pairs of STK orthologs are found in two Syne- 
chococcus strains, JA-2-3Ba and JA-3-3Ab, that share a close evolutionary relationship and are located at the bottom of $16 S$ rRNA tree, along with Gloeobacter violaceus PCC7421 (Fig. 1). STKs from Trichodesmium erythraeum IMS101 and Gloeobacter violaceus PCC7421 each form several separate clusters indicating obvious lineage-specific duplication events in these strains. Most kinase sequences from Anabaena and Nostoc are orthologs for their close evolutionary relationships, while the nonorthologous STK genes in Anabaena and Nostoc may have been produced by recent gene duplication or lateral transfer. This phylogenetic tree shows also that cyanobacterial STKs do not cluster strictly according to their structural characteristics, except for members of cbSTKIII which are clustered together in a big clade. Most STKs of cbSTKII do not cluster according to their additional domains, while some with specific additional domains, such as Pentapeptide and WD40, do cluster together (Fig. 4).

Thirty-eight STKs in cbSTKIII belong to a large cluster that also includes four STK proteins that do not contain HisKA and ATPase domains and therefore have been placed in other families. Tery_2857 and Tery_3863 have GAF domains and belong to cbSTKII, while Tery_2107 and Npun02008276 have only the STK domains and belong to cbSTKI-other (Fig. 4). To test the evolutionary relationships amongst the catalytic domains of dual protein kinases, their HiskA domains, and their GAF domains, a detailed analysis was performed by constructing three separate phylogenetic trees (Fig. 5). Only two groups of orthologs as defined by the catalytic domain (highlighted in Fig. 5) clustered together in the other two phylogenetic trees, indicating that two original genes were formed and appeared in the lineage prior to the divergence of Nostoc and Anabaena. Genes ancestral to orthologs that are conserved only in Anabaena PCC7120 and Anabaena ATCC29413 are assumed to have formed before the divergence of these two strains. Apparent incongruities may be explained by new recruitment or domain shuffling. For example, the HiskA domain of $N$. punctiforme gene Npun02001148 appears to have a distinct history from the HiskA domains of Anabaena PCC7120 All0323 and Anabaena ATCC29413 Ava_4716, but the three proteins share a common history with respect to their catalytic and GAF domains (see proteins highlighted in blue in Fig. 5) The second GAF domains in All3691, Ava_3596, Npun02004457, and Npun02004756 and the third GAF domain in Npun02004457 bear only a distant relationships with other STK GAF domains in cyanobacteria, including those in the same genes, indicating a recently recruitment. Alr0709 and Alr0710 lie adjacent to each other on the chromosome of Anabaena PCC7120 and show $75 \%$ similarities, indicating a recently gene duplication.

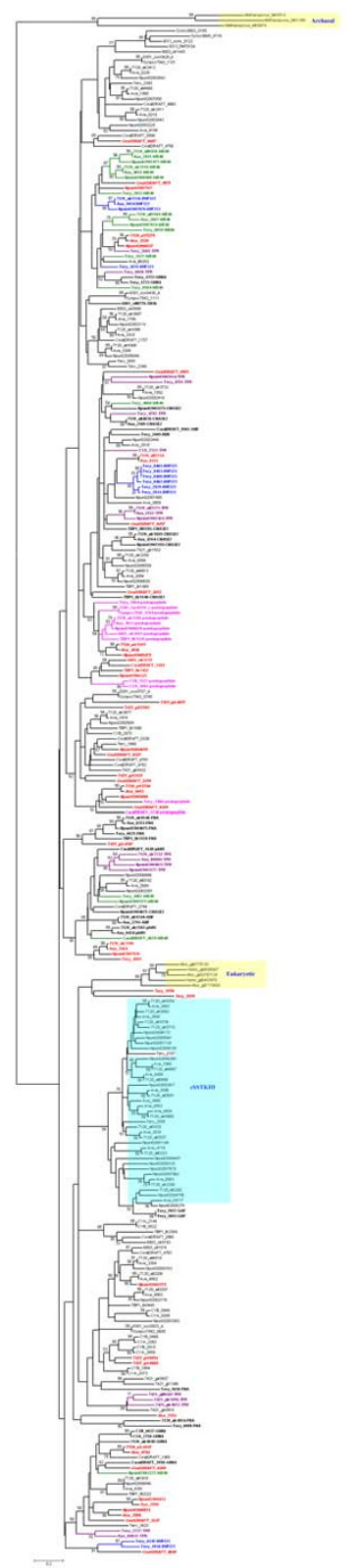

Figure 4

Phylogenetic analysis of the conserved catalytic domains of STKs. A phylogenetic tree based on the catalytic domains of cyanobacterial STKs was constructed as described in Methods. Strain names are as in Figure I. Bootstrap values $>50 \%$ are indicated on the branches. Additional domain names are also given following the gene names. STKs from subfamily cbSTKI-TM are marked in red and bold and those from cbSTKI-I are marked in red, bold, and italics. STKs with additional domains from cbSTKII are marked in bold and some major additional domains are marked in color: green WD40; blue DUF323; purple TPR; and magenta pentapeptide. Members of the family cbSTKIII family are highlighted in a cyan box. Archaeal and eukaryotic STK proteins are in yellow boxes. 

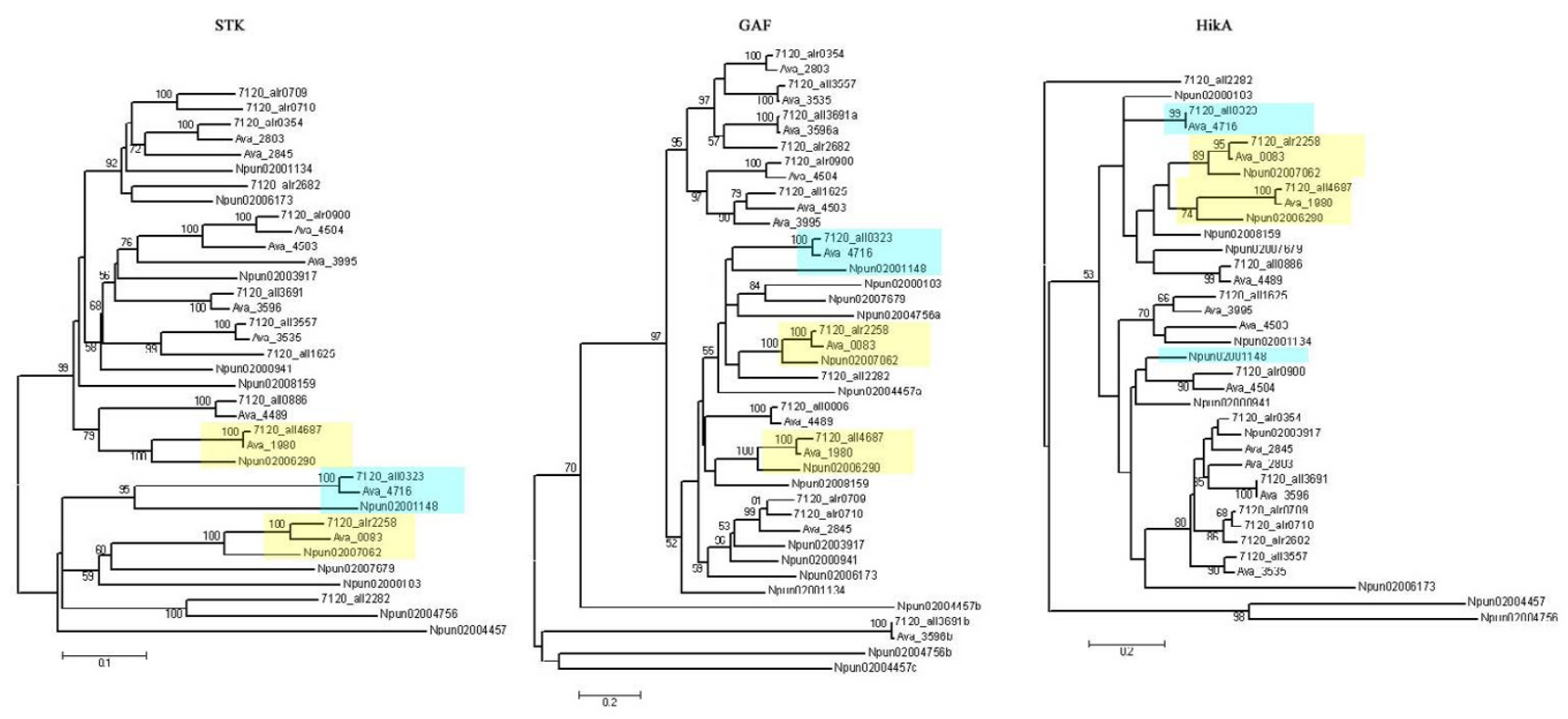

Figure 5

Phylogenetic analysis of STK catalytic domains, GAF and HikA additional domains. Phylogenetic trees were constructed from the amino acid sequences of the catalytic, GAF and HikA domains of dual protein kinases, as described in Methods. Branches with bootstrap support below 50\% have been collapsed. Four proteins (Anabaena PCC7I20_All369I,

A.variabilis_3596, N. punctiforme_02004756 and N. punctiforme_02004457) have two GAF domains and one (N.

punctiforme_02004457) has three. The strain abbreviations are as in Figure I. Orthologous genes, conserved in three strains, were marked in yellow box. Members in cyan boxes are possible examples of domain shuffling.

\section{Discussion}

Ser/Thr protein kinases, members of signal transduction systems, play important roles in responses to the environmental changes and intra-cellular signals [29]. This may be vital for their host cyanobacteria, which originated 2.5-3.5 billion years ago and exhibit the widest range of diversity in growth habitats of all photosynthetic organisms. Some of these kinases have demonstrated, in at least one case, possibly essential roles, illustrated by Sll0776 (SpkD) in Synechocystis PCC6803 which could not be completely knocked out [30].

The STK proteins used in this study were identified by Local Blast rather than from the COG database in IMG, and were manually checked to avoid false-negative and false-positive hits that commonly arise during large-scale automated analyses. Sequence alignment of conserved catalytic domains show that cyanobacterial STKs contain nearly the same subdomains as eukaryotic STKs but seem to be more conserved except in subdomain I. Eukaryotic STK proteins shared 12 nearly identical residues. Whereas a total of 15 highly conserved (shared by $>90 \%$ sequences) sites were found in cyanobacteria, seven of them were the same in eukaryotes. Such conserved motifs and amino acids may provide useful targets to evaluate their functions, and the high proportion of conserved res- idues shared by both eukaryotic organisms and cyanobacteria indicate a quite similar mechanism for domain organization and catalyzation.

Signal transduction systems provide basic mechanisms for cellular responses to environmental changes and connect environmental signals to cellular activities [29]. Thus different distributions of STKs through gene gain or loss may reflect various environmental selective pressures. Such as Synechococcus strains, which have similar genome size, hold different numbers of STK genes from zero to ten mainly for the different environmental conditions. Moreover, the number of STK genes in cyanobacteria is a function of the genome size, ecophysiology, and physiological properties of organism, as has been noted in general for bacteria $[46,47]$. STK genes are not ubiquitous in cyanobacteria. They are absent in four Prochlorococcus strains and one marine Synechococcus WH8102. Previous study showed that signal transduction and environmental stress response systems are dramatically reduced in marine unicellular Prochlorococcus and Synechococcus [48], both of whom live in the oligotrophic open ocean, and the major driving force behind might be a selective process favoring the adaptation of these cyanobacteria to adapt to the oligotrophic environment [49]. In contrast, filamentous heterocystous cyanobacteria, which differentiate heterocysts 
in response to the absence of combined nitrogen, and which display physiological and ecological properties including broad symbiotic competence with plants and fungi [41], showed a disproportionate number of STK genes.

Although Ser/Thr protein kinases were first discovered in eukaryotes and are evidently more widely dispersed amongst them, this is an insufficient basis to conclude that STKs originated in eukaryotes and were obtained by prokaryotes through lateral gene transfer. Zhang [50] and Leonard [51] have demonstrated that an ancestral protein kinase existed prior to the divergence of eucarya, bacteria, and archaea. The phylogenetic tree from our analysis shows STKs from archaea clustering at the root and cyanobacterial STKs clustering together with those from eukaryotes. The phylogenetic tree of cyanobacterial STKs, which was constructed by their conserved catalytic domains, is very complicated, and the distribution of STKs along the tree does not follow their domain structures or species phylogenies, indicating that frequent gene gain-and-loss events in cyanobacteria may obscure the actual relationships of STKs.

Besides gene gain-and-loss, novel proteins could also be generated by inserting or shuffling their domains. In these 21 cyanobacteria genomes, a total of 14 types of additional functional domains were found, much more than in other bacteria such as Mycobacterium and Streptomyces $[23,24]$. Additional domains are usually employed as sensor response modules [52-55] and can help organisms to assemble a sophisticated signal transduction apparatus. Their modularity may facilitate their spread into new genes and the rapid creation of novel functions [17]. Some additional domains (e.g. TPR) may have existed in the putative common ancestor of all life forms, and some (e.g. FHA) are thought to have been transferred from eukaryotes to bacteria [37]. Some prevalent additional domains, such as pentapeptides that are widely distributed in bacteria, may have been obtained from the ancestor at an early evolutional stage. The species-specific additional domains might have been recruited after speciation and duplicated recently, as in the example of the DUF323 domain in Trichodesmium erythraeum IMS101. Four STKs with DUF323 domains of T. erythraeum_06400643, which have high sequence similarities and neighbor on the chromosome, are closest paralogues and might have arisen from a recent duplication. STKs that cluster together according to their conserved catalytic domains do not always do so according to their additional domains, indicating the lateral recruitment of additional domains by STKs. A similar phenomenon was previously observed regarding the GAF and PAS domains found in PPM-family protein phosphatases in Streptomyces [56]. The two clusters with FHA and Pentapeptide domains are indicative of an early recruitment. GAF domains are prevalent in cyanobacterial two-component system and exist in all sequenced cyanobacterial genomes. But different STK orthologues did not recruit the same GAF and HiskA orthologs. It is assumed that HiskA and GAF domains were added to STK domains together and followed by domain shuffling. Orthologs that are conserved in three strains indicate their existence before the divergence of Anabaena and Nostoc, and the orthologs conserved in only two Anabaena strains suggest that they appeared before the divergence of the two Anabaena strains and after the split of Anabaena and Nostoc. Some sequences appear to have lost the additional domains, and some appear to have gained PAC and PAS domains, indicating that gene shuffling, deletion and insertion, as well as gene duplication, occurred during evolution.

Results from PPM-family protein phosphatases in Streptomyces, showed that PAS and GAF domains were clustered by physiological functions rather than taxonomic relationship [56]. So functions of substrate recognition in cyanobacterial STKs may be conferred largely by their additional domains too. For example, the CHASE (cyclase/histidine kinase-associated sensing extracellular) domain, always followed by three transmembrane regions, is present in more than one type of sensory proteins and can recognize several signals, such as cytokines and short peptides that are important for the development of an organism [57]. STKs, lacking transmembrane regions and acting as a receptor-like protein kinase in the cytoplasm, also appeared to have recruitted some additional domains such as FHA and GAF. The FHA domain is thought to play roles in a wide range of processes, including intracellular signal transduction, transcription, protein transport, DNA repair, and protein degradation [12]. The GAF domain family, linked to the binding of second messengers, is involved in cyclic nucleotide signaling, transcription, phototransduction, and probably many more unidentified processes [58]. Therefore, besides the conserved catalytic domain, the additional domains also play important roles in functions of kinases.

\section{Conclusion}

The availability of genome sequences provides a good opportunity for comparative analysis of gene families. Ser/Thr protein kinases have important effect on cyanobacteria living, as well as two component system. A total of 286 putative STK genes have been identified from 21 species of cyanobacteria using BlastP, TblastN and ClustalW, and 19 were not annotated originally as STKs. The number of STKs varied as a function of the genome size, ecophysiology and physiological properties. Fourteen types and 131 additional domains embedded in STK genes may assist their signal recognition functions. A similar catalytic mechanism was inherited with conventional 
conserved motifs, amino acids, and some uniquely cyanobacterial conserved residues. Gene duplication, loss, shuffling, insertion, and/or horizontal transfer appear to have played important roles during the evolution of cyanobacterial STKs.

\section{Methods}

Twenty-one species of cyanobacteria, including Prochlorococcus, Synechococcus, Synechocystis, Crocosphaera, Gloeobacter, Trichodesmium, Anabaena and Nostoc were used in this analysis. Since sequences of three Synechococcus strains RS9917, WH5701, WH7805 and one Prochlorococcus marinus strain MIT9312 are not yet fully released, they were not considered in our comparisons. All 21 genome sequences (as of Jan 2007) were accessed from IMG [59] in FASTA format. Four archaeal STK proteins in Methanopyrus kandleri AV19 were downloaded from the IMG database, and five eukaryotic STK proteins from human and mouse were downloaded from NCBI [60].

In order to identify genes that may encode STKs, a set of proven cyanobacterial STKs was used to search individual cyanobacterial genomes. The proven cyanobacterial STKs used were alr2502 (Pkn22) [40], alr4366 (PknA) [32], all4813 (PknC) [31], alr4368 (PknD) [20], alr3732 (PknE) [33] from Anabaena PCC 7120, and sll1574 (SpkA) [18], slr1697 (SpkB) [19], slr0599 (SpkC) [30], sll0776 (SpkD) [30] from Synechocystis PCC6803. Sll1574 (SpkA) is interrupted by an insertion element in the sequenced strain, and in this paper "Sll1574" refers to the reconstituted Sll1574-1575. BlastP and TblastN were conducted locally to search all proteins from each of the 21 cyanobacteria, using a threshold e-value of 1e-10. Proteins found by this method that fit the criteria for a genuine STK (see below) were added to the query set for another round of Blast searches. The procedure was continued until no new proteins were found.

Proteins identified by Blast were aligned by using ClustalW program [58] with a gap opening penalty of 10, a gap extension penalty of 0.2 , and Gonnet as the weight matrix. Alignment of query sets were examined by inspection of apparent Motifs I through XI per Hanks and Hunter [13] (Figure 2). A protein was accepted as an STK if it was possible to recognize the most conserved subdomains (i.e. II, V, VIb, VII, VIII, IX, and XI) and if those conserved amino acid residues known to participate in the function of STKs were present. Some minor deviations were tolerated, considered on a case-by-case basis. The ultimate decision was in the end subjective but in most cases quite clear cut.

Structure analyses of the obtained STKs were performed using the SMART $[27,61]$ and CDD (Conserved Domains Database) $[44,62]$ databases, relying on hidden Markov models and Reverse Position-Specific BLAST separately.
Sequences of the catalytic domains (about 280 aa in length) and additional domains used for phylogenetic tree construction were obtained from the SMART database and sequences with poor instances of Motif I and XI were checked manually. Trees based on 16s rRNA, STK conserved catalytic domains, GAF domains (mean of 150200 aa), and HiskA domains (mean of 65-100 aa) were constructed using NJ methods of the MEGA (Version 3.0) package [63], and the reliability of each branch was tested by 1000 bootstrap replications.

\section{Authors' contributions}

XWZ, FQZ and SQ devised the overall strategy for these studies. XWZ performed all database searches, acquired the sequence data, and prepared all figures and tables. XWZ and FQZ performed the conserved domain, structure, alignments, and phylogenetic analyses. XWZ, FQZ, YY, CWL and XYG jointly wrote the paper, and all authors have read and accepted the final version of the manuscript.

\section{Acknowledgements}

This work was supported by the Key Innovative Project of Chinese Academy of Science (KZCX2-YW-209, KZCX3-SW-223), Hi-Tech Research and Development Program (2006AA090303) of China, and the CAS/SAFEA International Partnership Program for Creative Research Teams (Research and Applications of Marine Functional Genomics).

\section{References}

I. Castenholz RW, Waterbury JB: Preface. Bergey's manual of systematic bacteriology. Williams and Wilkins, Baltimore 1989, 3:1710-1727.

2. Castenholz R: Species usage, concept, and evolution in the cyanobacteria (blue-green algae). J Phycol 1992, 28:737-745.

3. Stanier RY, Cohen-Bazire G: Phototrophic prokaryotes: the cyanobacteria. Annu Rev Microbiol 1977, 3 I:225-274.

4. Partensky F, Hess WR, Vaulot D: Prochlorococcus, a marine photosynthetic prokaryote of global significance. Microbiol Mol Biol Rev 1999, 63:106-127.

5. Parkinson JS: Signal transduction schemes of bacteria. Cell I993, 73:857-87I.

6. Stock JB, Stock AM, Motten JM: Signal transduction in bacteria. Nature 1990, 344:395-400.

7. Ulrich LE, Koonin EV, Zhulin IB: One-component systems dominate signal transduction in prokaryotes. Trends Microbiol 2005, 13:52-56.

8. Munoz-Dorado J, Inouye S, Inouye M: A gene encoding a protein serine/threonine kinase is required for normal development of M. xanthus, a gram-negative bacterium. Cell 1991, 67:995-1006.

9. Mann NH: Protein phosphorylation in cyanobacteria. Microbiology 1994, I40(Pt I2):3207-3215.

10. Kennelly PJ, Potts M: Fancy meeting you here: a fresh look at 'prokaryotic' protein phosphorylation. J Bacteriol 1996, I 78:4759-4764.

II. Kennelly PJ, Potts M: Life among the primitives: protein 0 phosphatases in prokaryotes. Front Biosci 1999, 4:D372-D385.

12. Zhang CC: Bacterial signalling involving eukaryotic-type protein kinases. Mol Microbiol 1996, 20:9-15.

13. Hanks SK, Hunter T: The eukaryotic protein kinase superfamily: kinase (catalytic) domain structure and classification. FASEB J 1995, 9:576-596.

14. Taylor SS, Knighton DR, Zheng J, Sowadski JM, Gibbs CS, Zoller MJ: A template for the protein kinase family. Trends Biochem Sci 1993, 18:84-89. 
15. Kennelly PJ: Archaeal protein kinases and protein phosphatases : insights from genomics and biochemistry. Biochem J 2003, 370:373-389.

16. Wang L, Sun YP, Chen WL, Li JH, Zhang CC: Genomic analysis of protein kinases, protein phosphatases and two-component regulatory systems of the cyanobacterium Anabaena sp. strain PCC 7120. FEMS Microbiol Lett 2002, 2 I 7:I55-I65.

17. Durochera D, Jacksonc SP: The FHA domain. FEBS Lett 2002, 5 I 3:58-66.

18. Kamei A, Yuasa T, Orikawa K, Geng XX, Ikeuchi M: A eukaryotictype protein kinase, SpkA, is required for normal motility of the unicellular cyanobacterium Synechocystis sp. strain PCC 6803. J Bacteriol 200I, I 83:I505-15I0.

19. Kamei A, Yoshihara S, Yuasa T, Geng X, Ikeuchi M: Biochemical and functional characterization of a eukaryotic-type protein kinase, SpkB, in the cyanobacterium Synechocystis sp. PCC 6803. Curr Microbiol 2003, 46:296-301.

20. Zhang CC, Libs L: Cloning and characterisation of the pknD gene encoding a eukaryotic-type protein kinase in the cyanobacterium Anabaena sp. PCC7I20. Mol Gen Genet 1998, 258:26-33.

21. Zhao F, Zhang X, Liang C, Wu J, Bao Q, Qin S: Genome-wide Analysis of Restriction-Modification System in Unicellular and Filamentous Cyanobacteria. Physiol Genomics 2006, 24:181-190.

22. Ashby MK, Houmard J: Cyanobacterial Two-Component Proteins: Structure, Diversity, Distribution, and Evolution. Microbiol Mol Biol R 2006, 70(2):472-509.

23. Av-Gay $Y$, Everett M: The eukaryotic-like Ser/Thr protein kinases of Mycobacterium tuberculosis. Trends Microbiol 2002 8(5):238-244.

24. Petrickova K, Petricek M: Review Eukaryotic-type protein kinases in Streptomyces coelicolor: variations on a common theme. Microbiol 2003, 149:1609-1621.

25. Krupa A, Srinivasan N: Diversity in domain architectures of Ser Thr kinases and their homologues in prokaryotes. $B M C$ Genomics 2005, 6: I29-149.

26. Zhang CC, Jiang JC, Sakr S, Wang L: Protein Phosphorylation on Ser, Thr and Tyr Residues In Cyanobacteria. J Mol Microbiol Biotechnol 2005, 9:।54-166.

27. Schultz J, Milpetz F, Bork P, Ponting CP: SMART, a simple modular architecture research tool: Identification of signaling domains. Proc Natl Acad Sci USA 1998, 95:5857-5864.

28. Skorko R: Polyphosphate as a source of phosphoryl group in protein modification in the archaebacterium Sulfolobus acidocaldarius. Biochimie 1989, 71:1089-1093.

29. Shi L, Potts M, Kennelly PJ: The serine, threonine, and/or tyrosine-specific protein kinases and protein phosphatases of prokaryotic organisms: a family portrait. FEMS Microbiol Rev 1998, 22:229-253

30. Kamei A, Yuasa T, Geng XX, Ikeuchi M: Biochemical Examination of the Potential Eukaryotic-type Protein Kinase Genes in the Complete Genome of the Unicellular Cyanobacterium Synechocystis sp. PCC 6803. DNA Res 2002, 9:71-78.

31. Gonzalez L, Phalip V, Zhang CC: Characterization of PknC, a Ser/Thr kinase with broad substrate specificity from the cyanobacterium Anabaena sp. strain PCC 7I 20. Eur J Biochem 200I, 268: I869-I875.

32. Zhang CC: A gene encoding a protein related to eukaryotic protein kinases from the filamentous heterocystous cyanobacterium Anabaena PCC 7120. Proc Natl Acad Sci USA 1993, 90: I 1840-। I844.

33. Zhang CC, Friry A, Peng L: Molecular and genetic analysis of two closely linked genes that encode, respectively, a protein phosphatase I/2A/2B homolog and a protein kinase homolog in the cyanobacterium Anabaena sp. Strain PCC 7I20. J Bacteriol 1998, 180:2616-2622.

34. Pratt WB: The hsp90-based chaperone system: involvement in signal transduction from a variety of hormone and growth factor receptors. Proc Soc Exp Biol Med 1998, 2 I 7:420-434.

35. Ho Y], Burden LM, Hurley JH: Structure of the GAF domain, a ubiquitous signaling motif and a new class of cyclic GMP receptor. EMBO J 2000, 1 9:5288-5299.

36. Joshi B, Janda L, Stoytcheva Z, Tichy P: PkwA, a WD-repeat protein, is expressed inspore-derived mycelium of Thermomonospora curvata and phosphorylation of its WD domain could act as a molecular switch. Microbiology 2000, I46:3259-3267.
37. Ponting CP, Aravind L, Schultz J, Bork P, Koonin EV: Eukaryotic signalling domain homologues in archaea and bacteria. Ancient ancestry and horizontal gene transfer. I Mol Biol 1999 , 289:729-745.

38. Larkin RM, Alonso JM, Ecker JR, Chory J: GUN4, a regulator of chlorophyll synthesis and intracellular signaling. Science 2003, 299:902-906.

39. Black K, Buikema WJ, Haselkorn R: The hglK gene is required for localization of heterocyst-specific glycolipids in the cyanobacterium Anabaena sp. strain PCC 7120. J Bacteriol 1995 I 77:6440-6448

40. Xu WL, Jeanjean R, Liu YD, Zhang CC: Pkn22 (alr2502) encoding a putative Ser/Thr kinase in the cyanobacterium Anabaena sp. PCC 7I20 is induced by both iron starvation and oxidative stress and regulates the expression of isiA. FEBS Lett 2003, 553: $179-182$

4I. Ohmori M, Ikeuchi M, Sato N, other I5 authors: Characterization of Genes Encoding Multi-domain Proteins in the Genome of the Filamentous Nitrogen-fixing Cyanobacterium Anabaena sp. Strain PCC 7I20. DNA Res 200I, 8:27|-284.

42. Phalip V, Li JH, Zhang CC: HstK, a cyanobacterial protein with both a serine/threonine kinase domain and a histidine kinase domain: implication for the mechanism of signal transduction. Biochem J 200I, 360:639-644.

43. Cheng Y, Li JH, Shi L, Wang L, Latifi A, Zhang CC: A Pairof IronResponsive Genes Encoding Protein Kinases with a Ser/Thr Kinase Domain and a His Kinase Domain Are Regulated by NtcA in the Cyanobacterium Anabaena sp. Strain PCC 7120. J Bacteriol 2006, 6:4822-4829.

44. Marchler BA, Bryant SH: CD-Search: protein domain annotations on the fly. Nucleic Acids Res 2004, 32(W):327-33I.

45. Woese CR, Kandler O, Wheelis ML: Towards a natural system of organisms: proposal for the domains Archaea, Bacteria, and Eucarya. Proc Natl Acad Sci USA 1990, 87:4576-4579.

46. Ashby MK: Survey of the number of two-component response regulator genes in the complete and annotated genome sequences of prokaryotes. FEMS Microbiol Lett 2004, 23 I:277-28I.

47. Galperin MY: A census of membrane-bound and intracellular signal transduction proteins in bacteria: bacterial IQ, extroverts and introverts. $B M C$ Microbiol 2005, 5:35-55

48. Dufresne A, Salanoubat M, Partensky F, other 18 authors: Genome sequence of the cyanobacterium Prochlorococcus marinus SSI 20, a nearly minimal oxyphototrophic genome. PNAS 2003, I00(I 7): 10020-10025.

49. Dufresne A, Garczarek L, Partensky F: Accelerated evolution associated with genome reduction in a free-living prokaryote. Genome Biol 2005, 6:RI4.

50. Han GN, Zhang CC: On the origin of Ser/Thr kinases in a prokaryote. FEMS Microbiol Let 200I, 200:79-84.

5I. Leonard C], Aravind L, Koonin EV: Novel families of putative protein kinases in bacteria and archaea: evolution of the "Eukaryotic" protein kinase superfamily. Genome Res 1998 , 8:1038-1047

52. Aravind L, Ponting CP: The GAF domain: an evolutionary link between diverse phototransducing proteins. Trends Biochem Sci 1997, 22:458-459.

53. Galperin MY, Nikolskaya AN, Koonin EV: Novel domains of the prokaryotic two-component signal transduction systems. FEMS Microbiol Lett 200I, 203: I I-2I.

54. Hofmann K, Bucher P: The FHA domain: a putative nuclear signalling domain found in protein kinases and transcription factors. Trends Biochem Sci 1995, 20:347-349.

55. Ponting CP, Aravind L: PAS: a multifunctional domain family comes to light. Curr Biol 1997, 7:R674-677.

56. Zhang WW, Shi L: Evolution of the PPM-family protein phosphatases in Streptomyces: duplication of catalytic domain and lateral recruitment of additional sensory domains. Microbiol 2004, I 50:4189-4197.

57. Zhulin IB, Nikolskaya AN, Galperin MY: Common Extracellular Sensory Domains in Transmembrane Receptors for Diverse Signal Transduction Pathways in Bacteria and Archaea. J Bacteriol 2003, 185:285-294.

58. Thompson JD, Higgins DG, Gibson TJ: CLUSTAL W: Improving the sensitivity of progressive multiple sequence alignment 
through sequence weighting, position-specific gap penalties and weight matrix choice. Nucleic Acids Res 1994, 22:4673-4680.

59. IMG Home [http://img.jgi.doe.gov/cgi-bin/pub/main.cgi]

60. NCBI Home [http://www.ncbi.nlm.nih.gov]

61. SMART Home [http://smart.embl-heidelberg.de/]

62. NCBI Conserved Domain Database (CDD) [http://] www.ncbi.nlm.nih.gov/Structure/cdd/cdd.shtml]

63. Kumar S, Tamura K, Nei M: MEGA3: Integrated software for molecular evolutionary genetics analysis and sequence alignment. Brief Bioinform 2004, 5:150-163.

Publish with Bio Med Central and every scientist can read your work free of charge

"BioMed Central will be the most significant development for disseminating the results of biomedical research in our lifetime. " Sir Paul Nurse, Cancer Research UK

Your research papers will be:

- available free of charge to the entire biomedical community

- peer reviewed and published immediately upon acceptance

- cited in PubMed and archived on PubMed Central

- yours - you keep the copyright

Submit your manuscript here:

http://www.biomedcentral.com/info/publishing_adv.asp
Biomedcentral 